\title{
Electrostatic Interactions of Asymmetrically Charged Membranes
}

\author{
-Dan Ben-Yaakov ${ }^{1}$, Yoram Burak ${ }^{2}$, David Andelman ${ }^{1,3}$ and S. A. Safran ${ }^{3}$ \\ Raymond and Beverly Sackler Faculty of Exact Sciences, Tel Aviv University, Ramat Aviv, Tel Aviv 69978, Israel \\ Kavli Institute for Theoretical Physics, University of California, Santa Barbara, CA 93106 USA \\ 3 Department of Materials and Surfaces, Weizmann Institute of Science, Rehovot 76100, Israel
}

PACS 82.70.-y - Disperse systems; complex fluids.

PACS 61.20.Qg - Structure of associated liquids: electrolytes, molten salts, etc.

\begin{abstract}
We predict the nature (attractive or repulsive) and range (exponentially screened or long-range power law) of the electrostatic interactions of oppositely charged and planar plates as a function of the salt concentration and surface charge densities (whose absolute magnitudes are not necessarily equal). An analytical expression for the crossover between attractive and repulsive pressure is obtained as a function of the salt concentration. This condition reduces to the high-salt limit of Parsegian and Gingell where the interaction is exponentially screened and to the zero salt limit of Lau and Pincus in which the important length scales are the inter-plate separation and the Gouy-Chapman length. In the regime of low salt and high surface charges we predict - for any ratio of the charges on the surfaces - that the attractive pressure is long-ranged as a function of the spacing. The attractive pressure is related to the decrease in counter-ion concentration as the inter-plate distance is decreased. Our theory predicts several scaling regimes with different scaling expressions for the pressure as function of salinity and surface charge densities. The pressure predictions can be related to surface force experiments of oppositely charged surfaces that are prepared by coating one of the mica surfaces with an oppositely charged polyelectrolyte.
\end{abstract}

Introduction. - The interactions between oppositely charged surfaces are important in both biological and materials science contexts. Proteins contain both cationic and anionic regions and in some cases, interactions between proteins are due to unlike charge attraction, mediated by the intervening counterions and salt. The delivery of cationic vesicles to cells - of interest in gene therapy applications - involves counterion and salt mediated electrostatic interactions of two oppositely charged membranes [1-3]. Similar considerations may also be important in understanding adhesion and fusion in systems of oppositely charged bilayers $[4,5]$. Recent experiments on hydrophobically prepared mica surfaces have indicated that such surfaces have domains with different charges and the observed long-range attractions (in the nanometer regime) may again be related to unlike charge attractions mediated by counterions and salt $[6,7]$.

In this paper we predict the interactions between two homogeneously charged surfaces with opposite charge. The surfaces are in aqueous solution that contains the counterions and added salt. The interactions can be attractive or repulsive, short-ranged (exponentially decaying) or long-ranged (power-law) depending on the ratio of the distance between the surfaces to the important length scales of the problem: (i) The Gouy-Chapman length, $\lambda_{\mathrm{GC}}=1 /\left(2 \pi l_{\mathrm{B}} \sigma\right)$ that is inversely proportional to the surface charge density $\sigma$; (ii) the Bjerrum length $l_{\mathrm{B}}=e^{2} / \varepsilon k_{\mathrm{B}} T$ equal to about $7 \AA$ in water $(\varepsilon \simeq 80)$ at room temperature; and, (iii) the Debye-Hückel (DH) length, $\lambda_{\mathrm{D}}=1 / \sqrt{8 \pi l_{\mathrm{B}} c_{b}}$ where $c_{b}$ is the bulk 1:1 salt concentration. Since there are several length scales there are several regimes that characterize the interactions.

In order to model interactions between charged surfaces, membranes and particles, one typically considers two planar surfaces separated by a distance, $d$. Previous studies considered the symmetric case where the two surfaces have fixed and equal surface charge densities. Within PoissonBoltzmann (PB) theory, it can be shown that the interaction between two surfaces with the same charge is always repulsive due to the counter-ion entropy. Other refinements include correction to the PB theory especially in the limit of strong surface charges and multi-valent counterions. The theory of the interactions between two surfaces with opposite charges has received less attention. The pioneering study of Parsegian and Gingell [14] considered high salt concentrations and used the linear DH theory, to 
predict regions of repulsive and attractive inter-plate pressure as a function of the distance and salt concentration. Lau and Pincus [13] considered the case of two oppositely charged surfaces with no added salt and found a simple analytical criterion for the crossover between attraction and repulsion.

The interesting, intermediate region of high surface charge and relatively low salt concentrations requires the use of the non-linear PB theory. There can be a large regime in which the distance between the plates is smaller than the DH screening length (which can be very large at low salt concentrations) but larger than the GouyChapman length (which can be very small for high surface charge) in which the non-linear treatment must be applied. The case of two plates with equal and opposite surface charges was considered recently by several authors $[15,16]$. In this case, where the two plates are electrically neutral and the ions in solution have no net charge, the force is always attractive. At some characteristic interplate distance, $d^{*}$, which scales as $\lambda_{\mathrm{GC}} \ln \left(\lambda_{\mathrm{D}} / \lambda_{\mathrm{GC}}\right)$, the counterions are released into the bulk reservoir provided that $\lambda_{\mathrm{GC}} \ll d^{*} \ll \lambda_{\mathrm{D}}$. In addition, for inter-plate separation in the range of intermediate values (between $\lambda_{\mathrm{D}}$ and $\lambda_{\mathrm{GC}}$ ), the pressure was shown to be long-ranged, and scales with the inverse of $d^{2}$ [15]. In the present work, we generalize these results and consider two oppositely charged surfaces whose charges are not necessarily equal in magnitude. This general case is important in order to analyze experiments in which surfaces are not completely antisymmetric. We use our analytical theory to predict a crossover between attraction and repulsion and the counterion release concept is extended to the asymmetric case. We also are able to consider several limiting regimes for the asymmetric case that complement the numerical solutions of the problem.

Poisson Boltzmann model. - The PoissonBoltzmann (PB) theory is a mean-field theory that relates the electric potential, $\psi(\vec{r})$, and the Boltzmann distribution for the ion number density, $c(\vec{r})$, at thermodynamic equilibrium. For two surfaces immersed in a 1:1 monovalent ionic solution, and for a dimensionless potential $\phi \equiv e \psi / k_{\mathrm{B}} T$, the $\mathrm{PB}$ equation reads:

$$
\nabla^{2} \phi=\kappa_{\mathrm{D}}^{2} \sinh \phi=\lambda_{\mathrm{D}}^{-2} \sinh \phi,
$$

where $\lambda_{\mathrm{D}}=\kappa_{\mathrm{D}}^{-1}$ is the Debye-Hückel (DH) screening length defined above.

We consider two charged surfaces that are infinite in extent in the $(x, y)$ plane and are separated in the $z$ direction by a distance $d$. In this case, the $\mathrm{PB}$ equation reduces to a one-dimensional equation in the coordinate $z$ perpendicular to the planes. The focus of this paper is on two oppositely charged plates that we call the asymmetric two plate problem. A positively charged plate with charge density $\sigma_{+}>0$ is located at $z=d / 2$, and a negatively charged one with $\sigma_{-}<0$ is located at $z=-d / 2$. While in the well-studied symmetric case, where $\sigma_{+}=\sigma_{-},[8]$ the potential (and ion densities) are symmetric about the midplane $z=0$, in the asymmetric case, the midplane is no longer a plane of symmetry. Instead, a separate boundary condition at each plate must be explicitly considered. These conditions relate the electric field at each plate to the surface charge density:

$$
\left.\frac{\mathrm{d} \phi}{\mathrm{d} z}\right|_{z= \pm d / 2}=4 \pi l_{\mathrm{B}}\left|\sigma_{ \pm}\right| \equiv \frac{2}{\lambda_{ \pm}}>0
$$

where $\lambda_{ \pm}$are the Gouy-Chapman lengths for the corresponding surfaces.

The spatial dependence of the potential and ion densities is obtained by solving the PB equation, eq. (1) subject to the boundary conditions, eq. (2). The profiles predict the local concentration of the mobile ions and their associated potentials; these can be measured, for example, using scattering techniques. However, more often, the forces exerted on the charged plates are measured [9]. For a given separation $d$, the pressure (or equivalently, the force per unit area) must be constant in the entire region between the plates if the system is in thermodynamic equilibrium. Thus, it is only necessary to calculate the pressure at any convenient point, $z$, within the gap. Because the two-plate system is in contact with a reservoir of mobile ions (the salt reservoir), the net pressure (in units of $k_{\mathrm{B}} T$ ) exerted on the plates is given by the difference between the inner and outer pressures, $\Pi=P_{\text {in }}-P_{\text {out }}$. This pressure can be calculated, for example, by integrating the $\mathrm{PB}$ equation once and relating the integration constant with the pressure $\Pi[8]$ :

$$
\Pi=-\frac{1}{8 \pi l_{\mathrm{B}}}\left(\frac{\mathrm{d} \phi}{\mathrm{d} z}\right)^{2}+2 c_{b}(\cosh \phi-1) .
$$

The $z$-independent osmotic pressure comprises two terms: (i) an attractive contribution whose origin is the electrostatic energy; this has the form of a negative term proportional to the square of the electric field [10] and (ii) a repulsive contribution that arises from the translational entropy of the ions and is given by the ideal-gas law. In this term we have already subtracted the outer pressure, $P_{\text {out }}=2 c_{b}$ as is explained above.

It is easy to show that the pressure for the case of symmetrically charged plates is always repulsive $(\Pi>0)$ within the $\mathrm{PB}$ approximation. However, in the general, asymmetric case the pressure can be either repulsive $(\Pi>0)$ or attractive $(\Pi<0)$ as we explain below.

Our two-plate problem is fully determined by four physical parameters: The two surface charge densities $\sigma_{ \pm}$, the ionic strength, $c_{b}$, and the separation $d$. However, by using normalized variables, it is easy to show that the problem is uniquely defined by three ratios: $\lambda_{ \pm} / \lambda_{\mathrm{D}}$ and $d / \lambda_{\mathrm{D}}$, where $\lambda_{ \pm}$is related to $\sigma_{ \pm}$in eq. (2) and $\lambda_{\mathrm{D}}$ is related to $c_{b}$. In our $\mathrm{PB}$ solution we use an alternative parametrization scheme including the dimensionless pressure $\hat{\Pi}=\Pi / c_{b}$, the surface potentials $\phi_{ \pm}=\phi( \pm d / 2)$. We can now relate those 
three parameters to the original ones by three relations. Two relations can be derived from eq. (3):

$$
\hat{\Pi}=2\left(\cosh \phi_{ \pm}-1\right)-\left(\frac{\lambda_{\mathrm{D}}}{\lambda_{ \pm}}\right)^{2} .
$$

Integration between the two boundaries, $\pm d / 2$, gives an additional, third relation:

$$
\frac{d}{\lambda_{\mathrm{D}}}=\int_{\phi_{-}}^{\phi_{+}} \frac{\mathrm{d} \phi^{\prime}}{\sqrt{2\left(\cosh \phi^{\prime}-1\right)-\hat{\Pi}}} .
$$

We can now express the PB solution and pressure $\hat{\Pi}$ [via eqs. (4) and (5)] as a function of the parameters that characterize the physical system namely, the surface charge densities, $\lambda_{+}, \lambda_{-}$, the DH length, $\lambda_{\mathrm{D}}$ (determined by the salt concentration), and the separation between the plates, $d$. For a few simplified cases, an analytical solution exists, while in the general asymmetric case we can write the potential, $\phi$, in terms of elliptic integrals [11] whose solution can be obtained only numerically. We next present a general analytical result that predicts when the interaction crosses over from repulsive to attractive as a function of the system parameters. The physical origin of this crossover is the competition between the electrostatic and entropic interactions described above.

Attractive to repulsive crossover. - The condition $\Pi=0$ in eq. (3) for the asymmetric, two plate system determines the cross-over from repulsive to attractive interactions in the system:

$$
-\frac{1}{8 \pi l_{\mathrm{B}}}\left(\phi^{\prime}\right)^{2}+2 c_{b}(\cosh \phi-1)=0 .
$$

This is a relation between the potential, $\phi(z)$, and its derivative $\phi^{\prime}$ at any point $z$. The condition, $\Pi=0$, also fixes one relation between the three dimensionless ratios: $\lambda_{ \pm} / \lambda_{\mathrm{D}}$ and $d / \lambda_{\mathrm{D}}$. Namely, this confines the system to a two dimensional surface in the three-dimensional parameter space $\left(\lambda_{+} / \lambda_{\mathrm{D}}, \lambda_{-} / \lambda_{\mathrm{D}}, d / \lambda_{\mathrm{D}}\right)$.

An analytical expression for this crossover is found by observing that the case of $\Pi=0$ can be exactly mapped onto the equations that describe a single plate in contact with the same reservoir [12]. For this purpose, we consider a system with a single, positively charged plate at $z=0$. The analytical expression for the potential and its derivative (electric field) are well known

$$
\begin{aligned}
\phi & =2 \ln \left(\frac{1+\gamma_{+} \mathrm{e}^{-z / \lambda_{\mathrm{D}}}}{1-\gamma_{+} \mathrm{e}^{-z / \lambda_{\mathrm{D}}}}\right) \\
\phi^{\prime} & =-\frac{1}{\lambda_{\mathrm{D}}} \frac{4 \gamma_{+} \mathrm{e}^{-z / \lambda_{\mathrm{D}}}}{1-\gamma_{+}^{2} \mathrm{e}^{-2 z / \lambda_{\mathrm{D}}}}
\end{aligned}
$$

where $\gamma_{+}=\sqrt{\left(\lambda_{+} / \lambda_{\mathrm{D}}\right)^{2}+1}-\left(\lambda_{+} / \lambda_{\mathrm{D}}\right)$. The mapping between the two problems is simply done by requiring that at distance $d$ away from the $z=0$ charged plate (the single plate case) the electric field is equal to the electric field as determined from Gauss's law at the negative plate located at $z=-d / 2$ in the two-plate problem: $\phi^{\prime}(d)=-2 / \lambda_{-}$. This results in the relation:

$$
\gamma_{+}=\mathrm{e}^{d / \lambda_{\mathrm{D}}} \gamma_{-}
$$

where $\gamma_{-}$is similarly defined as $\gamma_{-}=\sqrt{\left(\lambda_{-} / \lambda_{\mathrm{D}}\right)^{2}+1}-$ $\left(\lambda_{-} / \lambda_{\mathrm{D}}\right)$.

The relation (9) between $\gamma_{ \pm}$(or $\lambda_{ \pm}$) is equivalent to the $\Pi=0$ crossover in the asymmetric two plate system, between the repulsive, $\Pi>0$, and attractive, $\Pi<0$, regimes. When $\gamma_{-} \rightarrow 0$ (the negative plate is neutral, $\sigma_{-} \rightarrow 0$ ), the plates must repel each other. In addition, although we have only treated so far the case $\sigma_{+}>\left|\sigma_{-}\right|$, our results are quite general since the two-plate pressure is invariant under the exchange $\sigma_{+} \leftrightarrow\left|\sigma_{-}\right|$. Therefore, the condition for attraction reads:

$$
\mathrm{e}^{-d / \lambda_{\mathrm{D}}}<\frac{\gamma_{+}}{\gamma_{-}}<\mathrm{e}^{d / \lambda_{\mathrm{D}}},
$$

This result is plotted on fig. 1 where two lines separate a central region of attractive interactions from two wedges in the $\left(\sigma_{-}, \sigma_{+}\right)$plane, that denote repulsive interactions. In our plots, the charge densities are normalized by $\sigma_{D}=$ $1 / 2 \pi l_{\mathrm{B}} d$.

This result is exact for arbitrary salt concentration and surface charge densities. It has two limits that have been previously studied. One limit, that of zero salt, was analyzed by Lau and Pincus [13]. In this limit, the counterions in the solution balance the surface excess charge $\Delta \sigma=\sigma_{+}-\left|\sigma_{-}\right|$. Formally, we obtain this limit by taking $\lambda_{\mathrm{D}} \rightarrow \infty$ in eq. (10). Expanding $\gamma_{ \pm}$in powers of $\lambda_{ \pm} / \lambda_{\mathrm{D}}$, $\gamma_{ \pm} \simeq 1-\lambda_{ \pm} / \lambda_{\mathrm{D}}$, and eq. (10) yields:

$$
\left|\frac{1}{\sigma_{+}}-\frac{1}{\left|\sigma_{-}\right|}\right|<\frac{1}{\sigma_{d}}
$$

The crossovers from attraction to repulsion are plotted in fig. 1 for several salt concentrations using eq. (10) and the no-salt limit, eq. (11). The second, well-known limit of high salt concentrations, $\lambda_{\mathrm{D}} \ll \lambda_{ \pm}$, was studied by Parsegian and Gingell [14] in the 1970s by linearizing the $\mathrm{PB}$ equation. They derived the pressure in the linear $\mathrm{DH}$ regime. In this case, $\gamma_{ \pm}$can be approximated by $\gamma_{ \pm} \simeq$ $\lambda_{\mathrm{D}} / 2 \lambda_{ \pm}$in eq. (10), and the attraction condition is

$$
\mathrm{e}^{-d / \lambda_{\mathrm{D}}}<\frac{\sigma_{+}}{\left|\sigma_{-}\right|}<\mathrm{e}^{d / \lambda_{\mathrm{D}}}
$$

which reproduces the result of Parsegian and Gingell. In fig. 2 the line of zero pressure that separates attractive from repulsive interactions is plotted in the $\left(\sigma_{-} / \sigma_{+}, d / \lambda_{\mathrm{D}}\right)$ plane for three salt concentrations. The plot shows the high salt limit of Parsegian and Gingell [from eq. (12)], as well as an intermediate amount of salt $\left(\lambda_{+} / \lambda_{\mathrm{D}}=0.3\right)$, and a relatively low amount of salt $\left(\lambda_{+} / \lambda_{\mathrm{D}}=0.05\right)$ from eq. (10). 




Fig. 1: Regions of attraction and repulsion. $\sigma_{+}$and $\sigma_{-}$are the surface charge densities, and $\sigma_{d}=1 / 2 \pi l_{B} d$. The dashed and dash-dot lines represent the exact expression [eq. (10)] for crossover from attractive to repulsive interactions with $d / \lambda_{\mathrm{D}}=$ 1 and 2, respectively. The solid line shows the no-salt limit [eq. (11)].

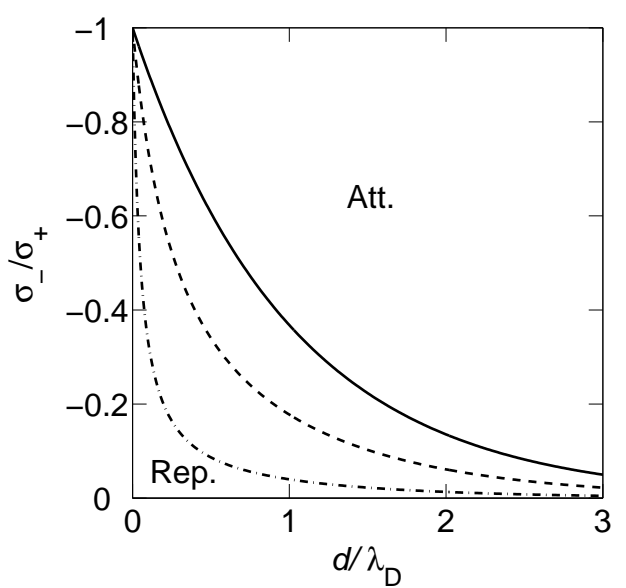

Fig. 2: The zero-pressure line $\Pi\left(\sigma_{-} / \sigma_{+}, d\right)=0$ in the $\left(\sigma_{-} / \sigma_{+}, d / \lambda_{\mathrm{D}}\right)$ plane. The dashed line represents the exact expression [eq. (10)] for crossover with $\lambda_{+} / \lambda_{\mathrm{D}}=0.3$, corresponding to $c_{b} \simeq 10^{-4} \mathrm{M}$ for typical mica effective net surface charge, $\sigma_{+} \simeq e / 4000 \AA^{2}$. On the dash-dot line $\lambda_{+} / \lambda_{\mathrm{D}}=0.05$, where for the same $c_{b} \simeq 10^{-4} \mathrm{M}$ the surface charge is $\sigma_{+} \simeq e / 670 \AA^{2}$. The crossover in the DH limit [eq. (12)] is shown in solid line.

We now compare the two figures and comment on the role of salt. In fig. 1 it is apparent that increasing the salt concentration enlarges the attractive region at the expense of the repulsive one. Another remark is that for $\left|\sigma_{ \pm}\right|>\sigma_{d}$ (large $d$ and/or strongly charged plates), the pressure is always negative (attraction) with no dependence on other system parameters like $\sigma_{-} / \sigma_{+}$and $\lambda_{\mathrm{D}}$. This is related to the asymptotic behavior of the no-salt crossover curves. 1

\footnotetext{
${ }^{1}$ Another observation is that in fig. 2 it looks like the predictive behavior is opposite as compared to fig. 1 because for larger amounts of salt the repulsive region is enlarged. However, the two figures are in total accord because in fig. 2 the $x$-axis is also scaled by $\lambda_{\mathrm{D}}$.
}

Gouy-Chapman pressure. - For the exact antisymmetric case $\left(\sigma_{+}=-\sigma_{-}\right)$and in the regime of low salt and strongly charged plates it was shown [15] that an approximate scaling relation between the pressure $\Pi$ and $d$ is: $\sqrt{c_{b} /|\Pi|} \ln \left(|\Pi| / c_{b}\right) \sim d / \lambda_{\mathrm{D}}$. The range of validity of this scaling relation is the diagonal ray in fig. 3 , $\sigma_{+} / \sigma_{d}=\left|\sigma_{-}\right| / \sigma_{d} \gg 1$, and in addition $d \ll \lambda_{\mathrm{D}}$. This result can be extended to the more general asymmetric region bounded by the hashed box in fig. $3: \sigma_{+} / \sigma_{d} \gg 1$ and $\left|\sigma_{-}\right| / \sigma_{d} \gg 1$. From the integral in eq. (5) the relation between the pressure and $d$ is deduced to be

$$
2 \sqrt{\frac{c_{b}}{|\Pi|}} \ln \left(\frac{4|\Pi|}{c_{b}}\right) \simeq \frac{d}{\lambda_{\mathrm{D}}}\left(1+\frac{\sigma_{d}}{\sigma_{+}}+\frac{\sigma_{d}}{\left|\sigma_{-}\right|}\right) .
$$

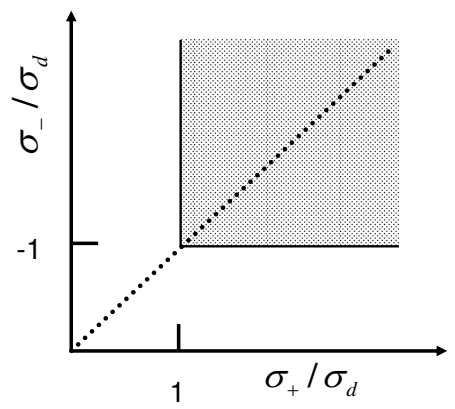

Fig. 3: The region of validity of the scaling relation is denoted by the hashed box in the $\left(\sigma_{+} / \sigma_{d}, \sigma_{-} / \sigma_{d}\right)$ plane. The dashed line denotes the anti-symmetric case.

We note that in the entire range of validity of this scaling expression, the right hand side of eq. (13) varies between $d / \lambda_{\mathrm{D}}$ and $3 d / \lambda_{\mathrm{D}}$, meaning that it is roughly described by the anti-symmetric result mentioned above [15]. Thus, as long as the plates are strongly charged, $\Pi$ does not depend on the surface charge densities. This relation can be solved iteratively for $\Pi(d)$ and the first iteration yields:

$$
\Pi \simeq-\frac{2}{\pi l_{\mathrm{B}} d^{2}} \ln ^{2}\left(d / 8 \lambda_{\mathrm{D}}\right) .
$$

Counter-ion release. - In the limit of infinite separation between the plates, an appropriate concentration of mobile cations and anions accumulate in the vicinity of each plate in order to neutralize the surface charge. When the plates are brought closer these two, oppositely charged clouds of mobile ions begin to overlap. Pairs of negative and positive counterions can thus neutralize each other and escape to the reservoir, where they gain entropy with no cost of electrostatic energy. This phenomenon of counterion release is the physical origin of the attractive forces between oppositely charged plates $[15,16]$. The parameter that characterizes the release of the counterions is defined as the excess charge per unit volume integrated over the entire separation between the surfaces:in eq. (10),

$$
\eta \equiv \int_{-d / 2}^{+d / 2} \mathrm{~d} z\left[c_{+}(z)+c_{-}(z)-2 c_{b}\right] .
$$


where $c_{ \pm}(z)$ are the number densities of the cations and anions. Safran [15] considered the relation between the pressure and the fraction of ions released into the reservoir for the exact antisymmetric case, $\left(\sigma_{+}=-\sigma_{-}\right)$, in the regime of low salt. Here we present a generalization of this result to the asymmetric case, where we focus on the same low salt, high surface charge regime but with $\Delta \sigma=\sigma_{+}-\left|\sigma_{-}\right|$which is small compared to $\sigma_{ \pm}$. Under these assumptions the integral in eq. (15) can be evaluated and gives:

$$
\eta \simeq 2 \sigma_{+}\left(1-\sqrt{2 \pi l_{\mathrm{B}} \lambda_{+}^{2}|\Pi|}\right)-\Delta \sigma,
$$

In the limit of $\Pi \rightarrow 0$ we find $\eta \simeq \sigma_{+}+\left|\sigma_{-}\right|$; this represents the largest possible value of excess counterions (beyond the bulk value $\left.\sigma_{b}=2 d c_{b}\right)$. In this limit, corresponding to large separations [eq. (14)] each of the surfaces is neutralized by its own cloud of counterions. As the separation between the two surfaces decreases, the pressure reaches its maximal attractive value $|\Pi| \rightarrow 1 / 2 \pi l_{\mathrm{B}} \lambda_{-}^{2}$, and the counterion excess approaches its minimal value: $\eta \simeq \Delta \sigma$. In this situation, not all the counterions are forced to remain in the gap between the plates. The two oppositely charged plates screen each other except for an excess of surface charge $\Delta \sigma$ that is compensated by the remaining counterions.

The largest attractive pressure occurs for a distance $d^{*}$ that can be estimated as $d^{*}=2 \lambda_{-} \ln 2 \lambda_{\mathrm{D}} / \lambda_{-}$. Note the difference in this situation between the asymmetric and the exact anti-symmetric case for which the pressure saturates at $d<d^{*}$.

Scaling regimes. - In the general asymmetric case we must consider the charge asymmetry ratio, $\lambda_{+} / \lambda_{-}$(or $\left.\left|\sigma_{-}\right| / \sigma_{+}\right)$as an additional parameter. We investigate several scaling regimes in the three-dimensional parameter space: $\left(\lambda_{+} / \lambda_{\mathrm{D}}, \lambda_{+} / \lambda_{-}, d / \lambda_{\mathrm{D}}\right)$. The pressure, as a function of the asymmetry ratio, can be treated by considering one of the following two limits: i) in the limit of $\left|\sigma_{-}\right| / \sigma_{+} \ll 1$, the negative plate can be taken as a neutral one, implying a repulsion between the plates, as demonstrated by eq. (10). In this limit, there is a mathematical correspondence between the asymmetric problem and the symmetric one $\left(\sigma_{+}=\sigma_{-}\right)$with about twice the surface separation, $d \rightarrow 2 d$. The symmetric configuration satisfies the boundary conditions corresponding to the case of one neutral and one charged surface by the vanishing of the electric field at the mid-plane. In this symmetric-like limit the pressure scales like the pressure in the symmetric case, as discussed in detail at ref. [8].

ii) On the other hand, when the surface charge densities are nearly equal (and opposite), i.e. $\Delta \sigma \ll\left|\sigma_{ \pm}\right|$, the pressure is attractive in a wide range of separations, and the formulae for the pressure are similar to those for the exact, antisymmetric case. Here, for simplicity, we present the regimes of the exact antisymmetric case with two oppositely charged plates, $\pm \sigma$. The different regimes are shown

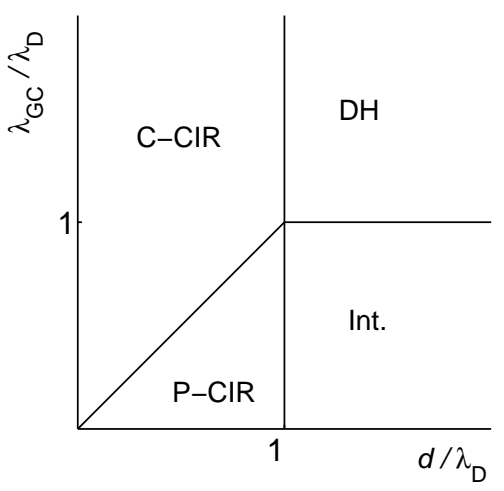

Fig. 4: A schematic view of the various limits of the PB equation for two oppositely charged plates (the anti-symmetric case). The four regimes discussed in the text are: Debye-Hückel (DH), Intermediate (Int.), Partial and Complete CounterIon Release (P-CIR and C-CIR). They are separated by four crossover lines: Int. $\leftrightarrow \mathrm{DH}$ at $\lambda_{\mathrm{GC}}=\lambda_{\mathrm{D}}$ and $\lambda_{\mathrm{D}}<d, \mathrm{DH} \leftrightarrow \mathrm{C}$ CIR at $\lambda_{\mathrm{D}}=d$ and $\lambda_{\mathrm{D}}<\lambda_{\mathrm{GC}}, \mathrm{C}-\mathrm{CIR} \leftrightarrow \mathrm{P}-\mathrm{CIR}$ at $d=\lambda_{\mathrm{GC}}$ and $\lambda_{\mathrm{D}}>d, \mathrm{P}-\mathrm{CIR} \leftrightarrow$ Int. at $\lambda_{\mathrm{D}}=d$ and $\lambda_{\mathrm{GC}}<\lambda_{\mathrm{D}}$.

in the $\left(\lambda_{\mathrm{GC}} / \lambda_{\mathrm{D}}, d / \lambda_{\mathrm{D}}\right)$ plane [fig. 4], and discussed below: (a) Debye-Hückel. The limit of $\lambda_{\mathrm{D}} \ll \lambda_{\mathrm{GC}}$ corresponds to low potentials $(\phi \ll 1)$ for which the PB equation can be linearized. We obtain an attractive pressure $(\Pi<0)$ as expected for two oppositely charged plates:

$$
\Pi \simeq-\frac{2}{\pi l_{\mathrm{B}} \lambda_{\mathrm{GC}}^{2}} \mathrm{e}^{-d / \lambda_{\mathrm{D}}} .
$$

This pressure expression decays exponentially with distance. (b) Complete Counter-ion Release. For small separations, $d \ll \lambda_{\mathrm{D}}$ and $d \ll \lambda_{\mathrm{GC}}$, the charge neutrality of the system is maintained by the surfaces, and all the cations and anions are released to the reservoir. This yields a direct electrostatic interaction of two capacitor plates of charge $\pm \sigma$ in a dielectric medium:

$$
\Pi \simeq-\frac{1}{2 \pi l_{\mathrm{B}} \lambda_{\mathrm{GC}}^{2}} .
$$

(c) Partial Counterion Release. This regime is defined by $\lambda_{\mathrm{GC}} \ll d \ll \lambda_{\mathrm{D}}$, where the plates are strongly charged and the salt concentration is low. Equation. (14) can be used to predict the pressure as a function of the separation, $d$. It is interesting to note that in this regime, the Gouy-Chapman pressure shows a long-range, power law dependence on $d$. It is also independent of the value of the surface charge, similarly to the result of Gouy-Chapman regime in the symmetric case. (d) Intermediate. When the plates are strongly charged, $\lambda_{\mathrm{GC}} \ll \lambda_{\mathrm{D}}$, the $\mathrm{PB}$ equation cannot be linearized. However, if the separation is large, $d \gg \lambda_{\mathrm{D}}$, the surfaces are weakly interacting and can be treated as two separated plates. As a result, the electric field at the midplane is given by summing these two contributions. Under these assumptions, the electric field can be approximated by that of a single plate, and 
the pressure is

$$
\Pi \simeq-\frac{8}{\pi l_{\mathrm{B}} \lambda_{\mathrm{D}}^{2}} \mathrm{e}^{-d / \lambda_{\mathrm{D}}} .
$$

In the $\mathrm{DH}$ and intermediate regimes the pressure decays exponentially with the scaled separation, $d / \lambda_{\mathrm{D}}$ due to screening effect of the salt ions. In the symmetric-like limit, the characteristic decay length is half as small. In the Gouy-Chapman regime the pressure is independent of the surface charge density, both in the antisymmetric and symmetric-like limits. This reveals a special property of the electrostatic interaction at medium-range separations: the length scales related to the surface charges, $\lambda_{ \pm}$and $\lambda_{\mathrm{D}}$, have no effect on the pressure (beside a small logarithmic correction as in eq. (14)). At small separations, the boundary conditions have the largest effect on the pressure between the plates. In the symmetric-like limit, the confinement of ions in between the plates results in a divergence of the pressure, whereas in the anti-symmetric like limit, the pressure saturates due to the complete release of all the counterions at small separations.

The similarity between the symmetric and antisymmetric cases can be seen at large and intermediate separations, while the distinction is evident at small separations. It is important to note that there is one major difference between these two limits: the symmetric-limit is purely repulsive, while the anti-symmetric one is always attractive.

Discussion. - The theoretical investigation in this work suggests that the asymmetric Poisson-Boltzmann model predicts several interesting physical regimes in the interaction of dissimilar charged bodies immersed in electrolyte solutions. In particular, the pressure dependence on the separation $\Pi(d)$ can be evaluated from both the scaling relations and the numerical solutions. These results can be tested in various experiments measuring forces between charged objects. One of the interesting results to be tested experimentally is the prediction for the crossover between attractive and repulsive interactions, $\Pi=0$, at high and low salt conditions. Others may include the crossover of the interactions from exponential decay to power law due to the onset of counterion release.

We wish to point out two features of the behavior at small $d$. The first is that in our model a diverging repulsive pressure is obtained in the limit of vanishing $d$ because of the assumption of fixed surface charge. However, effects such as charge regulation [17] and lipid demixing [18-20] can modify this assumption and may lead to to an overall attractive pressure for any $d$.

The second is that attractive van der Waals (vdW) interactions always prevail at small separations, $d<2 \mathrm{~nm}$ (in addition to the electrostatic interactions considered here). In experiments for small enough $d$, this vdW interaction is stronger than the electrostatic one and will overcome its repulsion. Thus, the only way to observe the pure electrostatic crossover between attractive and repul- sive interactions is to work in a set-up where this crossover occurs for a $d$ range beyond the influence of vdW interactions.

Acknowledgements. - We thank N. Kampf, J. Klein and S. Perkin for helpful discussion and for sharing with us their unpublished experimental results. One of us (DA) acknowledges support from the Israel Science Foundation (ISF) under grant no. 160/05, the US-Israel Binational Foundation (BSF) under grant no. 287/02, and a Weston visiting professorship award at Weizmann Institute where the latest part of this work was completed. SAS is grateful to the BSF and an EU Network grant for their support.

\section{REFERENCES}

[1] Koltover L., Salditt T., Rädler J. O. and Safinya C. R., Science, 281 (1998) 78.

[2] Wagner K., Harries D., May S., Kahl V., Rädler J. O. and Ben-Shaul A., Langmuir, 16 (2002) 303.

[3] Bruinsma R., Eur. Phys. J. B, 10 (1999) 175.

[4] Albersdörfer A., Feder T. and Sackmann E., Biophys. J., 73 (1997) 245; Bruinsma R., Behrisch A. and Sackmann E., Phys. Rev. E, 61 (2000) 4253.

[5] Hed G. and Safran S. A., Phys. Rev. Lett., 93 (2004) 138101.

[6] Perkin S., Kampf N. and Klein J., Phys. Rev. Lett., 96 (2006) 038301; Perkin S., Kampf N. and Klein J., J. Phys. Chem. B, 109 (2005) 3832.

[7] Meyer E., Lin Q., Hassenkam T., Oroudjev E. and Israelachvili J., PNAS, 102 (2005) 6839.

[8] Andelman D., in Handbook of Physics of Biological Systems, edited by Lipowsky R. and Sackman E., (Elsevier Science, Amsterdam) 1995, Vol. I, Chap. 12.

[9] Israelachvili J. N., Intermolecular and Surface forces (Academic, London) 1992, $2^{\text {nd }}$ ed.

[10] Landau L. D. and Lifshitz E. M., Electrodynamics of Continuous Media (Butterworth-Heinemann, Oxford) 1984, $2^{\text {nd }}$ ed.

[11] Behrens S. H. and Borkovec M., Phys. Rev. E, 60 (1999) 7040.

[12] Burak Y., Ph.D Thesis (Tel Aviv University) 2004, unpublished.

[13] Lau A. and Pincus P., Eur. Phys. J. B, 10 (1999) 175.

[14] Parsegian V. A. and Gingell D., Biophys. J., 12 (1972) 1192.

[15] Safran S. A., Europhys. Lett., 69 (2005) 826.

[16] Meier-Koll A. A., Fleck C. C. and von Grünberg H. H., J. Phys.: Condens. Matter, 16 (2004) 6041.

[17] Ninham B. W. and Parsegian V. A., J. Theor. Biol., 31 (1971) 405.

[18] Harries D., Podgornik R., Parsegian V. A., Mar-Or E. and Andelman D., J. Chem. Phys., 124 (2006) 224702.

[19] Haleva E., Ben-Tal N. and Diamant H., Biophys. J., 86 (2004) 2165.

[20] Russ C., Heimburg T. and von Grünberg H. H., Biophys. J., 84 (2003) 3730. 